\title{
Summary of Minutes of the GRAPPA Meeting Adjacent to the American Academy of Dermatology 67th Annual Meeting
}

\author{
WOLF-HENNING BOEHNCKE, ALICE B. GOTTLIEB, GERALD G. KRUEGER, ABRAR A. QURESHI, and AMIT GARG
}

\begin{abstract}
At a half-day meeting adjacent to the 67th annual meeting of the American Academy of Dermatology (AAD) in San Francisco, USA, in 2009, dermatology members of the Group for Research and Assessment of Psoriasis and Psoriatic Arthritis (GRAPPA) met to discuss recognition of psoriatic arthritis (PsA) in the dermatology clinic; multidisciplinary management of psoriasis patients; examples of physician tiering; comparative treatments for psoriasis and PsA; and biomarkers as predictors of response to treatment. Key results and minutes of the San Francisco meeting were presented at the 2009 GRAPPA annual meeting in Stockholm, Sweden, and are summarized here. (J Rheumatol 2011;38:553-6; doi:10.3899/jrheum.101120)
\end{abstract}

Key Indexing Terms:

PSORIATIC ARTHRITIS

PSORIASIS

SCREENING

GRAPPA

The Group for Research and Assessment of Psoriasis and Psoriatic Arthritis (GRAPPA) organized a half-day meeting adjacent to the 67th Annual Meeting of the American Academy of Dermatology (AAD) in San Francisco in 2009, as part of its efforts to facilitate a continuous interdisciplinary dialogue on the topic of psoriasis and psoriatic arthritis (PsA) among experts from different specialties. The discussion focused on (1) recognition of PsA in the dermatology clinic; (2) multidisciplinary management of the patient with psoriasis; and (3) several "hot topics," including physician tiering, comparative treatments for psoriasis and PsA, and biomarkers as predictors of response to treatment. Key results of the discussions at the San Francisco meeting are presented in Table 1 and summarized below. Because the discussion in San Francisco, primarily among dermatologists, provided a basis for further discussion and development of action items for the larger GRAPPA membership, which includes rheumatologists, radiologists, geneticists,

From the Department of Dermatology, Clinic of Johann Wolfgang Goethe University, Frankfurt am Main, Germany; Department of Dermatology, Tufts University School of Medicine and Tufts Medical Center, Boston, Massachusetts, USA; Department of Dermatology, University of Utah, Salt Lake City, Utah, USA; Department of Dermatology, Brigham and Women's Hospital, Harvard Medical School, Boston, Massachusetts, USA; and Department of Dermatology, Boston Medical Center, Boston University School of Medicine, Boston, Massachusetts, USA.

W.H. Boehncke, MD, Department of Dermatology, Clinic of the Johann Wolfgang Goethe University; A.B. Gottlieb, MD, PhD, Department of Dermatology, Tufts University School of Medicine and Tufts Medical Center; G.G. Krueger, MD, Department of Dermatology, University of Utah; A.A. Qureshi, MD, MPH, Department of Dermatology, Brigham and Women's Hospital, Harvard Medical School; A. Garg, MD,

Department of Dermatology, Boston Medical Center, Boston University School of Medicine.

Address correspondence to Dr. A. Garg, Department of Dermatology, Boston Medical Center, Boston University School of Medicine, 609 Albany Street, J207, Boston, MA 02118.E-mailagarg@bu.edu and epidemiologists, minutes of the San Francisco meeting were presented at the 2009 GRAPPA annual meeting in Stockholm, Sweden.

\section{Recognizing PsA in Dermatology Clinics}

Dr. Amit Garg (Department of Dermatology, Boston Medical Center, Boston University School of Medicine, Boston, MA) presented data from GRAPPA-IMPART (International Multicenter Psoriasis and Psoriatic Arthritis Reliability Trial), which measured the reliability of a comprehensive panel of skin and joint assessments among dermatologists and rheumatologists. The data suggested that trained dermatologists may be as reliable as rheumatologists in assessing tender joint count. The assessment of dactylitis, however, may be unreliable among dermatologists ${ }^{1}$. This presentation formed the basis of discussions concerning the following questions:

1. Should dermatologists have a role in recognizing the presence of PsA?

Dermatologists attending the meeting appreciated that the paradigm of managing patients with psoriasis may be shifting toward a multidisciplinary approach, and it is important that dermatologists remain attentively aware of comorbidities, especially the inherent risk of PsA.

Dermatologists acknowledged that the unpredictable, heterogeneous, and often insidious involvement of joints or juxtaarticular tendons and ligaments can make clinical recognition of PsA and distinction from other types of arthritis a challenge. It was agreed that in the absence of a diagnostic measure for PsA, the "gold standard" for diagnosis remains clinical assessment by the rheumatologist.

Attendees also agreed that dermatologists, as early caretakers of patients with psoriasis, might improve their patients' outcomes through early recognition of Personal non-commercial use only. The Journal of Rheumatology Copyright @ 2011 . All rights reserved. 
Table 1. Key questions and answers from the GRAPPA meeting adjacent to the 67th AAD meeting.

\begin{tabular}{|c|c|}
\hline Question & Answer \\
\hline Which methods would best allow dermatologists to recognize the presence of PsA? & $\begin{array}{l}\text { Physician evaluation, but this would require advanced training for the } \\
\text { dermatologist; also patient-reported screening tools }\end{array}$ \\
\hline What are the barriers to dermatologists recognizing the presence of PsA? & Clinical expertise; limited time; limited interest \\
\hline How shall we manage patients with severe psoriasis in the future? & $\begin{array}{l}\text { Take a comprehensive approach: Measure pulse, blood pressure, body } \\
\text { mass index, blood lipids, and blood glucose; assess joints }\end{array}$ \\
\hline What is the current perception on the mode of onset of methotrexate (MTX)? & $\begin{array}{l}\text { There may be a subgroup of psoriasis patients who may respond } \\
\text { quickly to relatively low doses ( } \leq 15 \mathrm{mg} / \text { week) of MTX }\end{array}$ \\
\hline What is the rationale for monitoring platelet activation in psoriasis patients? & $\begin{array}{l}\text { The platelet activation marker P-selectin may perform well as an } \\
\text { efficacy biomarker in the treatment of psoriasis }\end{array}$ \\
\hline
\end{tabular}

PsA and timely referral to and collaboration with a rheumatologist.

2. Which methods would best allow dermatologists to recognize the presence of PsA?

Members considered both physician evaluation and patient-reported symptoms as methods by which dermatologists may be able to recognize the presence of PsA.

It was acknowledged that while some dermatologists have specific interest and experience in evaluating patients for the presence of PsA, most would need further training in the assessment of joints and entheses. With training, the dermatologist may more reliably recognize PsA by appreciating disease demographics, asking the appropriate historical questions, performing simple targeted physical assessments, and utilizing laboratory and imaging studies.

Dermatologists said it would be helpful to have a practical framework for recognizing clinical features of PsA and distinguishing these features from other common forms of arthritis. When the CASPAR (Classification of Psoriatic Arthritis) criteria $^{2}$ were suggested, members believed most dermatologists would need specific training with regard to the presence of inflammatory arthritis, but that identification of the remaining components of the CASPAR criteria was probably more achievable. Members also were interested in assessing the reliability of CASPAR criteria in the dermatology clinic, and it was emphasized that the CASPAR criteria were developed for the purpose of classification and not as a diagnostic tool. Several dermatologists commented that these criteria might be difficult to use in the setting of a private practice.

Patient-reported screening tools enabling dermatologists to recognize the presence of PsA were also discussed. The most helpful examples were those developed by the groups of Dafna Gladman (ToPAS), Abrar Qureshi (PASE), and Philip Helliwell (PEST) ${ }^{3,4,5}$; discussions of these screening questionnaires are included in this supplement ${ }^{6,7,8}$. Availability of these validated questionnaires might have a profound effect on the ability of dermatologists to recognize PsA early in the course of the disease.
3. What are the barriers to dermatologists recognizing the presence of PsA?

It was acknowledged that expertise, time, and possibly interest in joint and entheseal assessments are limitations to recognition of PsA by general dermatologists.

\section{Managing Comorbidities in Psoriatic Patients}

Dr. Wolf-Henning Boehncke (Department of Dermatology, Clinic of Johann Wolfgang Goethe University, Frankfurt am Main, Germany) led a discussion regarding the particular relevance of longterm cardiovascular comorbidity management in patients with severe psoriasis 9,10 . This topic was extensively discussed in San Francisco, further represented at the Stockholm meeting, and is summarized elsewhere in this supplement ${ }^{11}$.

\section{Physician Tiering}

Dr. Alice Gottlieb (Tufts University School of Medicine and Tufts Medical Center, Boston, MA) led a discussion on the current state of the "Clinical Performance Improvement Initiative in Massachusetts" and its potential implications for patients with psoriasis and PsA as well as for the dermatologists and rheumatologists who care for them. The Massachusetts Group Insurance Commission, with an interest in promoting cost-effective care, is tiering physicians according to costs associated with their diagnostic and therapeutic care for patients. It is important to note that tiering criteria are not based on relevant patient health outcomes and tiering algorithms do not take patient severity into account; also, the validity of the tiering methodology has not been independently assessed. Nevertheless, physician tiering threatens both physicians and patients. Physicians with expertise in psoriasis and PsA often utilize costly biologic therapies for patients with severe disease that requires complex management. In Massachusetts, these patients are threatened with significantly higher copayments, which may dissuade them from consulting physicians with the expertise they need. Similarly, physicians may be hesitant to treat these sicker patients appropriately because of the threat of 
receiving a bad tiering score that would discourage all patients from returning to them.

There was consensus that this paradigm may be duplicated in other US states, and comparable approaches were reported from other countries. For example, German physicians who substantially increase costs by prescribing expensive drugs, compared with costs for average physicians in the same specialty, may be forced to compensate payers out of their own pocket. Thus, physicians with severely affected patients who need higher-priced drugs may be stigmatized as "expensive" doctors or even monetarily punished for their practice, which may cause them to minimize their care of severely sick patients or decrease their use of effective but costly medications in order to maintain their practice. In the end, severely affected patients may have decreased access to the very physicians who can treat them appropriately.

\section{Lessons from CHAMPION}

Dr. Gerald Krueger (Department of Dermatology, University of Utah, Salt Lake City, UT) reviewed comparative trials, specifically a head-to-head comparison between methotrexate (MTX; conventional therapy) and adalimum$\mathrm{ab}$ (biologic) for induction therapy of moderate to severe plaque-type psoriasis (CHAMPION study) ${ }^{12}$. The primary finding was that adalimumab was superior to MTX. There was consensus that comparative studies are of major importance particularly in developing treatment recommendations as part of evidence-based guidelines.

However, more detailed analyses of the CHAMPION study suggested that among the patients treated with MTX, a subgroup of patients showed quicker responses on a low dose of MTX. Thus, careful subanalyses of patients treated with conventional systemic antipsoriatic drugs may be important in future clinical research, with a goal of defining patients with a high likelihood of responding readily to such drugs. This would be particularly important for MTX, because it is still widely used for psoriasis/PsA, although often demonstrating a slower onset of efficacy.

Dr. Krueger also discussed the use of polyglutamination as a means to differentiate between potentially good and poor responders to MTX therapy ${ }^{13}$. The rationale is that polyglutamination represents a key step in the mode of action of MTX in inflammatory diseases. The process of polyglutamination of MTX promotes a sustained build-up of adenosine, which appears to have antiinflammatory/immune suppressive activity. Clinical data are contradictory, however: in rheumatoid arthritis, "good glutaminators" responded better to MTX than "bad glutaminators," while in psoriasis, there is currently no convincing evidence of such an association. This lack of evidence, however, could simply be a matter of less than optimum assessment, because the early report had a very limited number of subjects.

Finally, Dr. Krueger mentioned 2 studies that suggest it may be possible to predict both efficacy and toxicity of MTX in patients with psoriasis, based on single-nucleotide polymorphisms in the MTX efflux transporters $\mathrm{ABCC} 1$ and ABCG2. This aspect is still a matter of current research.

\section{P-Selectin as an Efficacy Biomarker in the Treatment of Psoriasis}

Dr. Boehncke reported on a recent cross-sectional study ${ }^{14}$ where P-selectin expression is a readily measurable activation marker on platelets with known pathogenetic relevance as an effector mechanism in inflammation. With regard to cutaneous inflammation, its involvement in leukocyte extravasation as an integral step in the development of an inflammatory infiltrate has been documented. In an attempt to validate platelet P-selectin expression as a possible biomarker for inflammation, it was prospectively investigated in more than 200 patients with psoriasis and other inflammatory skin conditions. On the day of admission, P-selectin was expressed on significantly more platelets in samples from patients with inflammatory skin diseases compared to healthy controls. P-selectin expression was significantly reduced under effective treatment of the respective disorder. Of note, a highly significant correlation between the Psoriasis Area and Severity Index and P-selectin $(r=0.51$, $\mathrm{p}<0.000001$ ) was observed in the subgroup of patients with psoriasis. Moreover, platelet $\mathrm{P}$-selectin showed a significant correlation with C-reactive protein $(r=0.46, p=0.00008)$. Finally, it was shown that measuring soluble P-selectin using a commercial enzyme-linked immunosorbent assay yielded results that again correlated well with flow cytometry of membrane-bound P-selectin expressed on platelets $(r=0.63, p<0.01)$. Overall, this study provides evidence that plasma P-selectin may be a valid biomarker to assess the efficacy of antipsoriatic therapy.

\section{Further GRAPPA Dermatology-related Meetings}

The series of GRAPPA meetings in conjunction with international conferences on dermatology will continue in Gothenburg, Sweden, on the occasion of the 2010 annual meeting of the European Academy of Dermatology and Venereology.

\section{REFERENCES}

1. Chandran V, Gottlieb A, Cook RJ, Duffin KC, Garg A, Helliwell P, et al. International multicenter psoriasis and psoriatic arthritis reliability trial for the assessment of skin, joints, nails, and dactylitis. Arthritis Rheum 2009;61:1235-42.

2. Helliwell PS, Taylor WJ. Classification and diagnostic criteria for psoriatic arthritis. Ann Rheum Dis 2005;64 Suppl 2:ii3-8.

3. Gladman DD, Schentag CT, Tom BD, Chandran V, Brockbank J, Rosen C, et al. Development and initial validation of a screening questionnaire for psoriatic arthritis: the Toronto Psoriatic Arthritis Screen (ToPAS). Ann Rheum Dis 2009;68:497-501.

4. Husni ME, Meyer KH, Cohen DS, Mody E, Qureshi AA. The PASE questionnaire: Pilot-testing a psoriatic arthritis screening and evaluation tool. J Am Acad Dermatol 2007;57:581-7.

$$
\text { Personal non-commercial use only. The Journal of Rheumatology Copyright (c) 2011. All rights reserved. }
$$


5. Ibrahim GH, Buch MH, Lawson C, Waxman R, Helliwell PS. Evaluation of an existing screening tool for psoriatic arthritis in people with psoriasis and the development of a new instrument: the Psoriasis Epidemiology Screening Tool (PEST) questionnaire. Clin Exp Rheumatol 2009;27:469-74.

6. Chandran V, Gladman DD. Toronto Psoriatic Arthritis Screening (ToPAS) Questionnaire: A report from the GRAPPA 2009 annual meeting. J Rheumatol 2011;38:546-7.

7. Dominguez P, Husni ME, Garg A, Qureshi AA. Psoriatic Arthritis Screening and Evaluation (PASE) questionnaire and the role of dermatologists: A report from the GRAPPA 2009 annual meeting. J Rheumatol 2011;38:548-50.

8. Helliwell P. Psoriasis Epidemiology Screening Tool (PEST): A report from the GRAPPA 2009 annual meeting. J Rheumatol 2011;38:551-2.

9. Boehncke WH, Boehncke S, Schon MP. Managing comorbid disease in patients with psoriasis. BMJ 2010;340:b5666.

10. Kimball AB, Gladman D, Gelfand JM, Gordon K, Horn EJ, Korman NJ, et al. National Psoriasis Foundation clinical consensus on psoriasis comorbidities and recommendations for screening. J Am Acad Dermatol 2008;58:1031-42.
11. Boehncke WH, Gladman DD, Chandran V. Cardiovascular comorbidities in psoriasis and psoriatic arthritis: pathogenesis, consequences for patient management, and future research agenda: a report from the GRAPPA 2009 annual meeting. J Rheumatol 2011;38:567-71.

12. Saurat JH, Stingl G, Dubertret L, Papp K, Langley RG, Ortonne JP, et al. Efficacy and safety results from the randomized controlled comparative study of adalimumab vs. methotrexate vs. placebo in patients with psoriasis (CHAMPION). Br J Dermatol 2008;158:558-66.

13. Warren RB, Chalmers RJ, Griffiths CE, Menter A. Methotrexate for psoriasis in the era of biological therapy. Clin Exp Dermatol 2008;33:551-4

14. Garbaraviciene J, Diehl S, Varwig D, Bylaite M, Ackermann H, Ludwig RJ, et al. Platelet P-selectin reflects a state of cutaneous inflammation: possible application to monitor treatment efficacy in psoriasis. Exp Dermatol 2010;19:736-41. 